\title{
One Kind of Control Method Based on PLC of Stepper Motor
}

\author{
Yujie Chong ${ }^{\mathrm{a}}$, Changjie $\mathrm{Xu}^{\mathrm{b}}$, Haimei Feng ${ }^{\mathrm{c}}$, \\ Xin Shi ${ }^{\mathrm{d}}$, Jie $\mathrm{Li}^{\mathrm{e}}$, \\ Chang Dư ${ }^{\mathrm{f}}$, Junhong Wang ${ }^{\mathrm{g}}$ \\ NCIST YanJiao, San He HeBei Province, China \\ a505480036@qq.com, ${ }^{\mathrm{b}} 729511692 @ q q . c o m,{ }^{\mathrm{c}} 76358265 @ q q . c o m$, \\ d543505993@qq.com, e491923668@qq.com, \\ f416863719@qq.com, ${ }^{\mathrm{g}} 2589427992 @ q q . c o m$
}

\begin{abstract}
This paper discussed a method of PLC controlling motor. Including the start and stop, forward and inversion of motor, Positive \&negative switching in the process of Running. And realizing low speed, high speed, medium speed and switch operation, accurate control of step motor number. it was elaborated the programming block diagram and ladder diagram of the implementation status in detail.
\end{abstract}

Keywords-PLC; stepping motor; ladder diagram.

\section{THE INTRODUCTION}

Due to good control and accurate localization features of the stepper motor, it is widely used in accurate location, such as CNC machine, drawing machine, steel machine, automatic control computing device, such as automatic recording instrument automatic control field.

PLC as a simplified computer, has fully functional, flexible, general, control system simple, cheap characters. It can modify the program. The volume of PLC is small. Hardware maintenance is convenient and the price is cheap.PLC are widely used all over the world. It brings great benefits for production and life. Therefore, by studying the use of PLC to control the step motor, can achieve precise positioning control, and can reduce the control cost, and maintenance. Before stepping motor need to rely on the drive to control, with the continuous development of technology is perfect, PLC has directly by its output pulse stepper motor function, so it is helpful for accurate control of stepping motors.

\section{THE DESIGN OF THE PLC PROGRAM}

\section{A. The connecting way of PLC and drive}

There are two main connection ways between stepper motor and PLC: one is the stepper motor at both ends of the wire winding to the output points of PLC, as shown in figure 1, by writing program control electricity, each coil in a certain order to achieve positive \&negative; Another way is to use PLC high-speed pulse output (PLS), received the circular distributor, again by annular pulse distributor of output control the movement of the stepper motor, including the speed and direction, as shown in figure 2. The former requires more dots, programming more troublesome, not suitable for high speed stepping motor; The latter only need two dots, a pulse supply, a control steering, easy to use, simple wiring, easy programming.

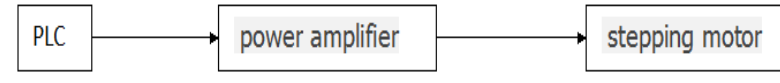

Fig.1. PLC and stepping motor directly connected

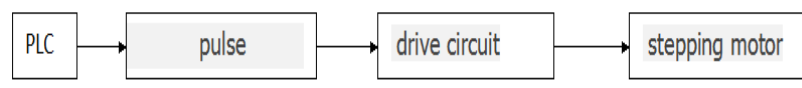

Fig.2. PLC and stepping motor connected with drive circuit

\section{B. Three-phase six stepper motor control}

For three phase six take control of the stepper motor, mainly in three aspects: on and off of the three-phase winding of sequence control, step motor stepper speed control steps and control. Forward order: $B C$ - C - A - AB - B - CA - A; Reverse order: AC - C - A - CB - B - BA - A; Each step from the Angle of speed; Pulse frequency and the number.

Can around these a few respects, can make the following specific requirements:

(1) May be started forward and inversion.

(2) The operation process, positive \&negative kept switching from time to time;

(3) Every start from A phase operation;

(4) The stepper motor can start properly, and can achieve high speed, medium speed and low speed three gear control, and can be controlled variable speed;

(5) Steps can realize the control of step motor;

\section{CONTROL PROGRAM BLOCK DIAGRAM}

Controlled by the specific requirements, can make a step motor in starting the program diagram of run time, as shown in figure 3 . By a diagram of the work as the fundamental basis, consider to the specific requirements of the control, the first ladder diagram program can be divided into four programming module, the module 1 : stepper motor step speed selection (low speed, medium speed and high speed); Module 2: displacement shift register; Module 3: three-phase circular distributor; Module 4: pulse generator; Module 5: A, B, C three-phase winding object control; Module 6: steps of stepper motor control. Then, connect each module, and finally after debugging, perfect, solid control requirements. 


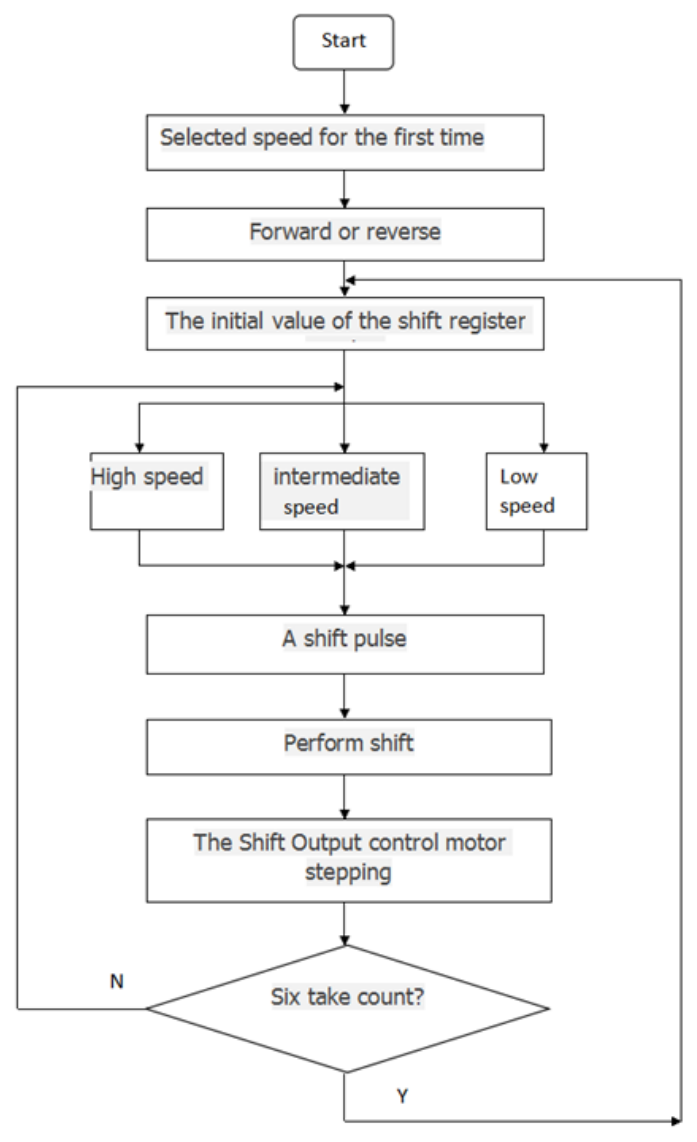

Fig.3. stepper motor control program block diagram

IV. LADDER DIAGRAM PROGRAMMING

\section{A. I/O addressing}

Control stepping motor each input switch and control A, B, C three-phase winding work output in the PLC I/O addressing as shown in table 1.

TABLE 1. INPUT/OUTPUT ADDRESSING

\begin{tabular}{|c|c|c|c|}
\hline Port & Input & Port & Output \\
\hline SB0 & Start I0.0 & Q0.0 & A phase \\
\hline SB1 & Low speed I0.1 & Q0.1 & B phase \\
\hline SB2 & Intermediate speed I0.2 & Q0.2 & C phase \\
\hline SB3 & High speed I0.3 & & \\
\hline SB4 & Forward or reverse I0.4 & & \\
\hline SB5 & Stop I1. I & & \\
\hline SB6 & Single Step I0.5 & & \\
\hline SB7 & Ten step I0.6 & & \\
\hline SB8 & Hundreds of step I0.7 & & \\
\hline
\end{tabular}

\section{B. State the truth table}

Using shift instruction to stepping control. First designated shift register MB0 (8), according to the three phase six steps taken in order, the initial value of the shift register are shown in table 2 .
TABLE 2 SHIFT REGISTER INITIAL VALUE

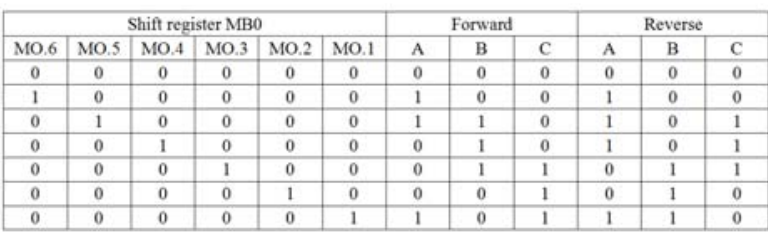

To draw a three-phase winding control logic relation:

(1) The forward

A phase $\mathrm{Q} 0.0=\mathrm{M} 0.6+\mathrm{M} 0.5+\mathrm{M} 0.1$

$\mathrm{B}$ phase $\mathrm{Q} 0.1=\mathrm{M} 0.4 \mathrm{M} 0.5++\mathrm{M} 0.3$

Phase C Q0.2 = M0.2 M0.3 + + M0.1

(2) The reversal

A phase Q0.0 = M0.6 + M0.5 + M0.1

$\mathrm{B}$ phase $\mathrm{Q} 0.1=\mathrm{M} 0.2 \mathrm{M} 0.3++\mathrm{M} 0.1$

Phase C Q0.2 = M0.4 M0.5 + + M0.3

C. Ladder diagram program and their comments

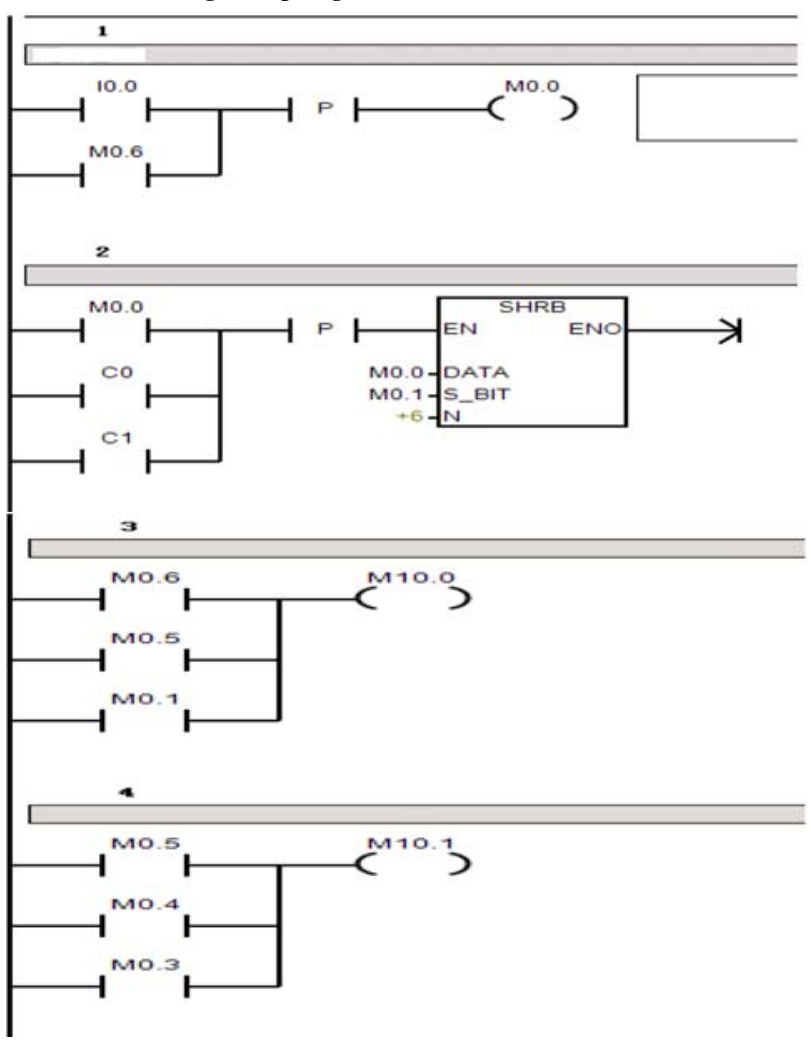




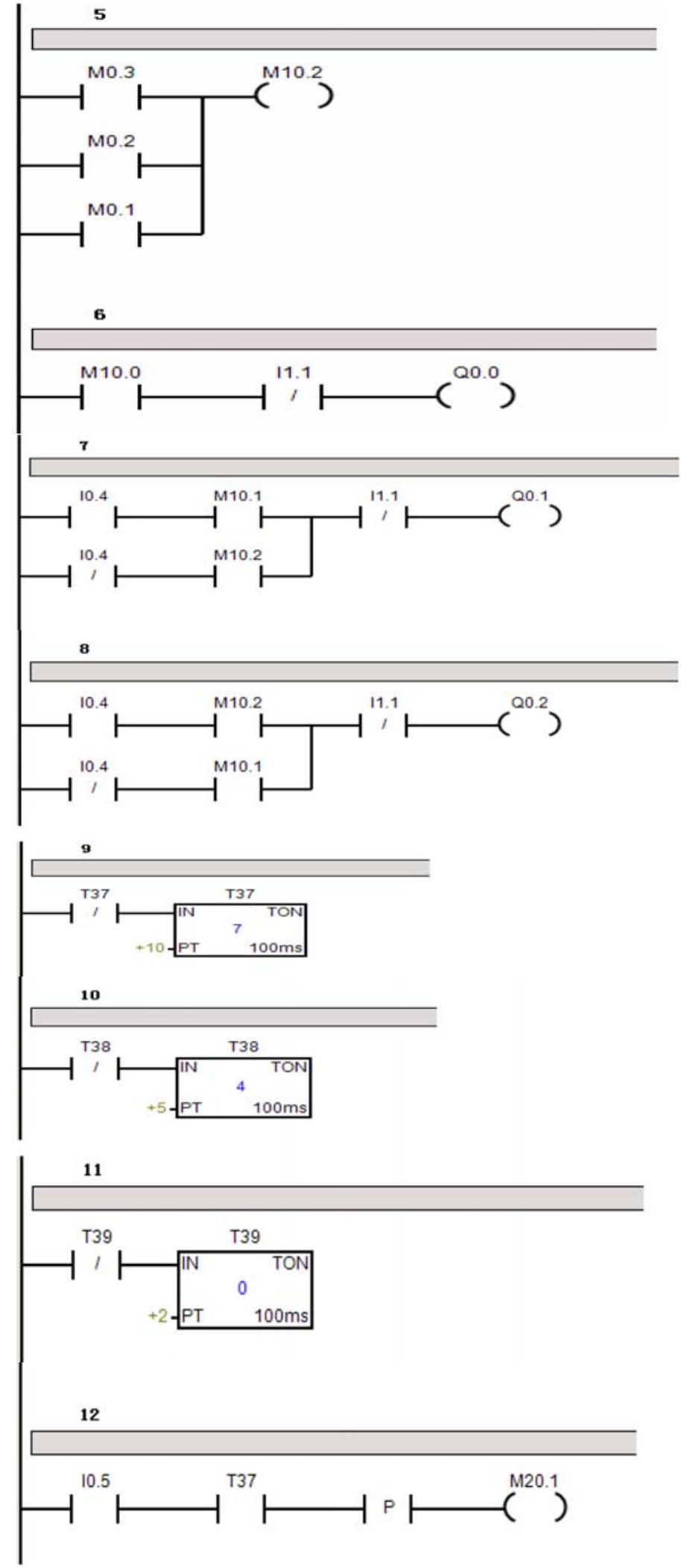

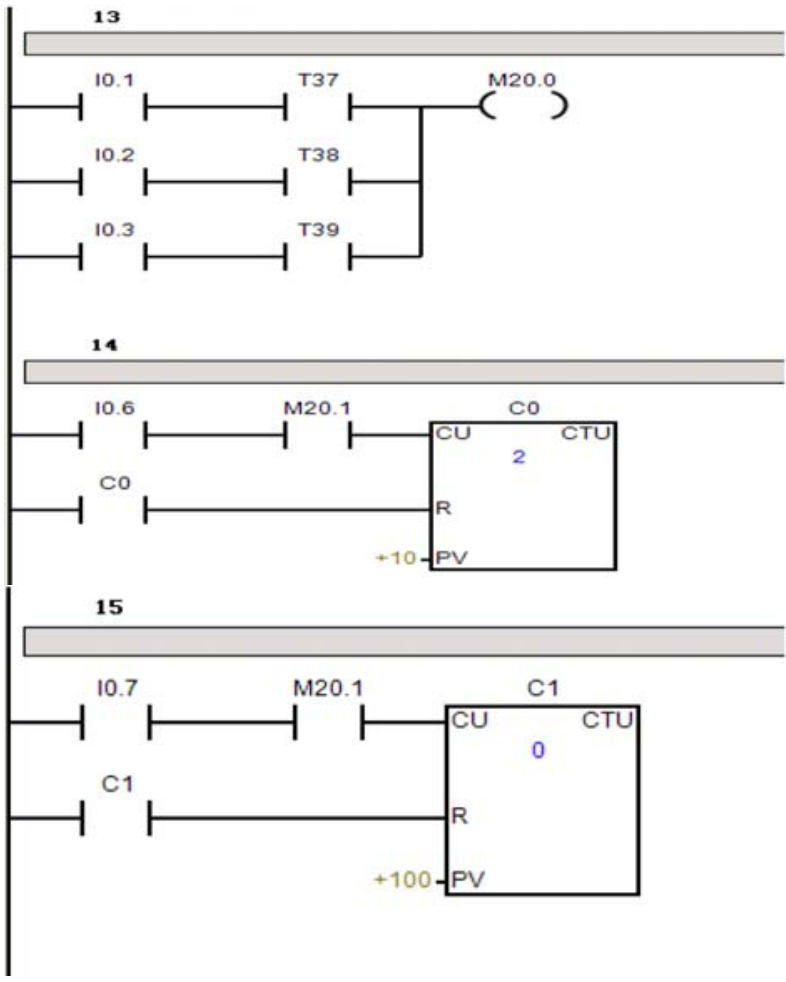

Ladder diagram to explain

Networks 1: assignment of startup for displacement registers its lowest 1; To the highest level displacement register $\mathrm{M} 0$ again for the most low fu 1;

Internet 2: M0.0 most data input, S_BIT to register its lowest, moving six consecutive to the left operation; CO to control the operation of 10 steps; C1 to control the operation of 100 steps;

Network 3-5: according to table 3 logical relationship, for three phase six circular distributor;

Network 6-8: the output signal to realize, is the positive \&negative drive (three-phase output);

Network: September 11 by timer pulse transmitter (T37 for slow, T38 for medium speed, T39 for rapid), is the pulse generator;

Network 12: single-step operation;

Network 13: pulse controller;

Network 14 to 15: steps by steps set of control;

\section{SUMMARY AND OUTLOOK}

This article will stepper motor at both ends of the wire winding to the output points of PLC, controlled by program each coil electricity, according to certain order to achieve positive \&negative, realize low speed, high speed, medium speed and switch operation; A precise control of stepping motor steps, write out the implementation program block diagram and the implementation process. We hope the precise trajectory control will be studied and discussed in the future 


\section{REFERENCES}

[1] Core and Attribute Reduction Algorithms Based on Compatible Discernibility Matrix[A]. Proceedings of the 2009 Internation al Conference on Computational Intelligence and Natural Com puting(Volume 2)[C]. 2009

[2] A Classification Algorithm Based on Simplified Fuzzy Rules B ase[A]. Proceedings of 2010 Third International Symposium o n Knowledge Acquisition and Modeling (KAM 2010)[C]. 201 0

[3] Simplified Virtual Instrument Design Based on Mini Interface [A]. Proceedings of 2010 First International Conference on Ce llular,Molecular Biology, Biophysics and Bioengineering(Volum e 1)[C]. 2010

[4] Simplified Support Vector Machine Method for QRS Wave Det ection[A]. Proceedings of 2009 IEEE 10th International Confe rence on Computer-Aided Industrial Design \& Conceptual Des ign[C]. 2009

[5] V. V. Athani.Stepper Motors: Fundamentals Applications and D esign. . 2007

[6] Paul Acamley.STEPPING MOTORS:a guide totheory and practi ce. . 2002

[7] D.O. Carrica,,S.A.Gonzez,M.Benedetti.A high speed velocity con trol algorithm of multiple stepper motors. Mechatronics . 200 4

[8] El-Sharkawi, M.A,Yonghong Guo.Adaptive fuzzy control of a b elt-driven precision positioning table. IEEE International Confe rence on Electric Machines and Drives. 2003

[9] Petar Crnosiga,Branislav Kuzmanovic,Stipe Ajdukovic.Microcom puter implementation of optimalalgorithms for closed-loop con trol of hybrid stepper motor drives. IEEE Trans. IndustrialEle ctronics. 2000 